Portland State University

PDXScholar

Chemistry Faculty Publications and

Presentations

Chemistry

$9-4-2003$

\title{
Pressure-induced insulating state in an organic superconductor
}

\author{
Gary L. Gard \\ Portland State University \\ Javid Mohtasham \\ J. A. Schlueter \\ C. Pfleiderer \\ J. Wosnitza
}

See next page for additional authors

Follow this and additional works at: https://pdxscholar.library.pdx.edu/chem_fac

Part of the Chemistry Commons, and the Physics Commons

Let us know how access to this document benefits you.

\section{Citation Details}

Hagel, J., Wosnitza, J., Pfleiderer, C., Schlueter, J. A., Mohtasham, J., \& Gard, G. L. (2003). Pressureinduced insulating state in an organic superconductor. Physical Review B, 68, 104504.

This Article is brought to you for free and open access. It has been accepted for inclusion in Chemistry Faculty Publications and Presentations by an authorized administrator of PDXScholar. Please contact us if we can make this document more accessible: pdxscholar@pdx.edu. 


\section{Authors}

Gary L. Gard, Javid Mohtasham, J. A. Schlueter, C. Pfleiderer, J. Wosnitza, and J. Hagel 


\title{
Pressure-induced insulating state in an organic superconductor
}

\author{
J. Hagel, ${ }^{1}$ J. Wosnitza, ${ }^{1}$ C. Pfleiderer, ${ }^{2}$ J. A. Schlueter, ${ }^{3}$ J. Mohtasham, ${ }^{4}$ and G. L. Gard ${ }^{4}$ \\ ${ }^{1}$ Institut für Festkörperpkysik, Technische Universität Dresden, D-01062 Dresden, Germany \\ ${ }^{2}$ Physikalisches Institut, Universität Karlsruhe, D-76128 Karlsruhe, Germany \\ ${ }^{3}$ Materials Science Division, Argonne National Laboratory, Argonne, Illinois 60439, USA \\ ${ }^{4}$ Department of Chemistry, Portland State University, Portland, Oregon 97207, USA
}

(Received 10 March 2003; published 4 September 2003)

\begin{abstract}
The electronic-transport properties of the quasi-two-dimensional organic superconductor $\beta^{\prime \prime}$-(BEDT-TTF) $)_{2} \mathrm{SF}_{5} \mathrm{CH}_{2} \mathrm{CF}_{2} \mathrm{SO}_{3}$, where BEDT-TTF stands for bisethylenedithio-tetrathiafulvalene, have been investigated in magnetic fields up to $15 \mathrm{~T}$ and under hydrostatic pressure up to about 14 kbars. Shubnikov-de Haas data reveal a nonmonotonic pressure dependence of the holelike Fermi surface, a roughly linear increase of the electron $g$ factor, and an approximately linear decrease of the cyclotron effective mass. By assuming that the latter reflects the pressure-induced reduction of the superconducting coupling parameter $\lambda$ the rapid reduction of the superconducting transition temperature $T_{c}(p)$ can be reasonably well described by the modified McMillan equation. Above about $12 \mathrm{kbars}$ the material becomes insulating with an activated resistive behavior. This first-order metal-insulator transition has a hysteresis of about $3 \mathrm{kbars}$. This unexpected behavior is assumed to be of structural origin, although clear changes of electronic band-structure properties precede the phase transition.
\end{abstract}

DOI: $10.1103 / P h y s R e v B .68 .104504$

PACS number(s): 74.70.Kn, 71.18.+y, 74.62.Fj

\section{INTRODUCTION}

One of the distinguished properties of organic chargetransfer salts is their large variety of different and often competing ground states. Key parameters to control these sensitive phases are external magnetic field and pressure. Thereby, in the usual naive picture the application of pressure leads to an enlarged electronic-band overlap resulting in an increase in dimensionality, whereas a magnetic field effectively reduces the dimensionality by forcing the electrons to move in trajectories perpendicular to the field. Indeed, the quasi-onedimensional (1D) electronic structure of the so-called Bechgaard salts ${ }^{1}$ is susceptible to a Peierls transition leading to a low-temperature insulating state at ambient pressure. Only by applying a sufficient pressure the metallic state survives and superconductivity may be observed. This has led to the discovery of the first organic superconductor. ${ }^{2}$ The extraordinarily rich pressure-field-temperature phase diagram of the Bechgaard salts, which has been studied extensively, is basically understood taking into account the electronic bandstructure topology. ${ }^{1}$

Equally sensitive to external pressure are the quasi-twodimensional (2D) organic charge-transfer salts. For these no generic phase diagram - as for the Bechgaard salts-exists, since the different crystal structures lead to diverse groundstate properties. Anyway, in case an insulating state exists at ambient pressure a metallic or finally superconducting state often can be realized by applying sufficient pressure. Both in 1D and 2D systems a further increase of pressure usually leads to a rapid suppression of superconductivity. The decrease of the superconducting transition temperature $T_{c}$ with applied pressure is extraordinarily large and may reach up to $\mathrm{d} T_{c} / d p \approx-3.8 \mathrm{~K} / \mathrm{kbars}$ for the $2 \mathrm{D}$ organic superconductor $\kappa$-(BEDT-TTF $)_{2} \mathrm{Cu}(\mathrm{NCS})_{2}$, where BEDT-TTF stands for bisethylenedithio-tetrathiafulvalene (or ET for short). ${ }^{3,4}$

The origin of the strong pressure effect on $T_{c}$ in organic superconductors is not fully understood, but phenomenologically it is caused either by a decreasing density of states at the Fermi level as a result of an increasing electronic bandwidth or by a reduced interaction potential between the Cooper pairs. Both effects lead to a reduced coupling strength $\lambda$ causing $T_{c} \propto \exp (-1 / \lambda)$ to decrease. ${ }^{5} \mathrm{Up}$ to date it is discussed controversially whether the Cooper-pair coupling is phonon mediated as in conventional superconductors or whether unconventional interactions, such as antiferromagnetic correlations, are responsible for the attractive forces. (For recent reviews, see Refs. 6, 7.) In favor for the latter scenario magnetic interactions have been found in some organic charge-transfer salts leading to antiferromagnetic insulating ground states. On the other side, the existence and importance of electron-phonon interaction in organic superconductors is clearly established and may well account for the pairing interaction. ${ }^{6,7}$ In this context the strong pressure dependence of $T_{c}$ may easily be explained by the pronounced softness of the crystalline bonds in organic materials and a corresponding drastic change of phonon modes under pressure.

In a number of previous experimental studies the pressure dependence of selected band-structure parameters has been investigated for different 2D organic conductors. ${ }^{8-15}$ Thereby, however, in many cases the measurements were restricted to one fixed angle $\Theta$ of the magnetic field with respect to the normal of the conducting BEDT-TTF planes, ${ }^{8-13}$ or only selected superconducting and bandstructure parameters were reported. ${ }^{14,15}$ Only rarely have attempts been made to extract the correlation between $T_{c}$ and the coupling parameter $\lambda . .^{9,10,13}$

Here, we present a comprehensive investigation of the pressure dependence (up to about 14 kbars) of the electronictransport, superconducting, and band-structure properties of the 2D organic charge-transfer salt $\beta^{\prime \prime}$-(BEDT-TTF) $)_{2} \mathrm{SF}_{5} \mathrm{CH}_{2} \mathrm{CF}_{2} \mathrm{SO}_{3}$. At ambient pressure, 
specific heat yields a bulk $T_{c}=4.4 \mathrm{~K}$ for this superconductor, ${ }^{16}$ whereas resistive data give $T_{c}=5.2 \mathrm{~K}$ from the point where the resistance has dropped by $50 \%$ (resistive midpoint, see below). ${ }^{17}$ In previous hydrostaticpressure studies up to about $2.3 \mathrm{kbar}, d T_{c} / d p$ $=-1.34 \mathrm{~K} / \mathrm{kbar}$ was determined by use of ac-susceptibility measurements. ${ }^{4}$ This fits with thermal-expansion results that gave a similar initial hydrostatic-pressure dependence of $T_{c} \cdot{ }^{18}$ For this material no other pressure-dependent properties have been reported so far.

The experimental investigation of possible pressure-induced phase transitions in $\beta^{\prime \prime}$-(BEDT-TTF) ${ }_{2} \mathrm{SF}_{5} \mathrm{CH}_{2} \mathrm{CF}_{2} \mathrm{SO}_{3}$ is especially motivated by the fact that slight modifications of the anion immediately destroy superconductivity and even lead to insulating ground states. ${ }^{19,20}$ Although the origin of this behavior is unclear, charge ordering might be a driving mechanism. This might either be caused by the largely different electronegativities of the participating ions in the anion layer or by a large intersite Coulomb repulsion in the band-electron system. The latter has been predicted to occur for the $\beta^{\prime \prime}$ structure based on the theoretical study of the quarter-filled extended Hubbard model on a square lattice. ${ }^{21}$ As a further consequence, based on this model $d$-wave superconductivity mediated by charge fluctuations has been proposed, ${ }^{22}$ but not verified experimentally. Here, the application of pressure is an obvious tool to influence sensitively the electronic interactions, to test the theoretical predictions, and to yield valuable information on the electronic properties in these charge-transfer salts.

\section{EXPERIMENT}

Single crystals of $\beta^{\prime \prime}$-(BEDT-TTF) ${ }_{2} \mathrm{SF}_{5} \mathrm{CH}_{2} \mathrm{CF}_{2} \mathrm{SO}_{3}$ were grown by the standard electrocrystallization technique. ${ }^{23}$ The interplane resistances of two samples were measured by a two-point (sample 1) and four-point (sample 2) method inside a $\mathrm{CuBe}$ pressure cell by use of a lowcurrent ac-resistance bridge. The current leads of $50 \mu \mathrm{m}$ (sample 1) or $25 \mu \mathrm{m}$ (sample 2) gold wire were glued with graphite paste to the samples. The results for both samples are basically identical except for the remnant resistance of about $20 \Omega$ in the superconducting state of sample 1 caused by the contact resistances in the two-point configuration. In the following, we present the resistance data only for sample 2 , but include the results of sample 1 when discussing the pressure dependences of $T_{c}$ and the band-structure parameters (Fig. 6 below).

The dimensions of the used CuBe pressure cell $(12 \mathrm{~mm}$ diameter, $27 \mathrm{~mm}$ length) enabled an in situ rotation around two axes inside of a ${ }^{3} \mathrm{He}$ cryostat that was equipped with a superconducting 15-T magnet. The samples were mounted in such a way that the normal to the conducting ET plane was aligned by eye along the cylinder axis of the pressure cell. This alignment was achieved only to within a few degrees caused by the limited sample space and a sample movement during the initial pressure application. The latter can hardly be avoided. Nevertheless, the well-known anisotropies of the superconducting critical fields, which are maximal when the magnetic field $B$ is aligned parallel to the ET planes, and of

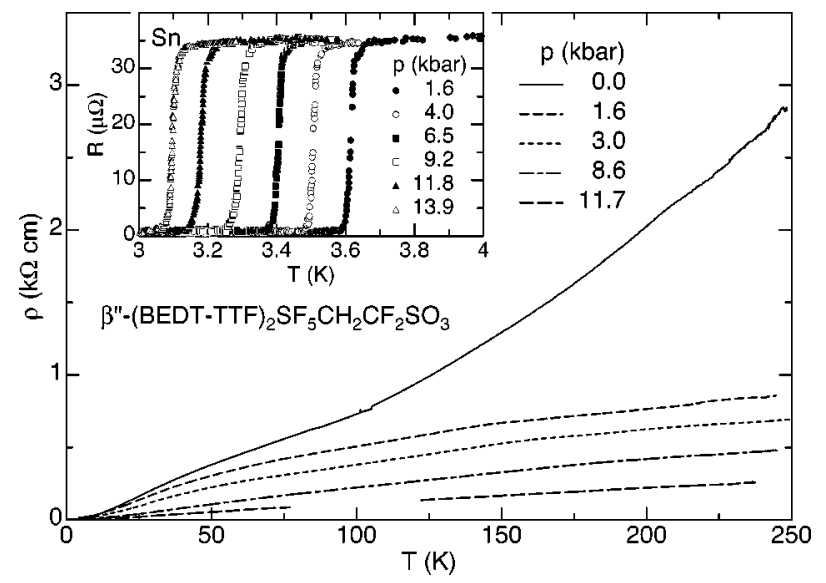

FIG. 1. Temperature dependence of the resistivity of sample 2 for different selected pressures in the metallic state. The inset shows some resistive superconducting transitions of the Sn gauge used to determine the pressures at low temperatures.

the 2D Fermi surface, leading to the $1 / \cos \Theta$ dependence of the quantum-oscillation frequency (see below), allowed a precise assignment of the sample orientation. During the course of the experiments, the relative orientation of the samples within the pressure cell remained fixed independent of successive pressure changes performed at room temperature outside of the cryostat.

The used pressure medium, a mineral oil (GKZh-94), and the slow cooling of the pressure cell over many hours ensured a quasiisotropic pressure distribution over the whole temperature range. The data at zero pressure were obtained with the pressure medium removed. In spite of the small cell dimensions pressure of up to $\sim 14$ kbars at low temperatures could be applied reproducible. Thereby, the pressures were determined at low temperatures with an accuracy of about 0.2 kbars by measuring resistively the superconducting transition of a small Sn wire mounted next to the samples inside of the pressure cell. $T_{c}$ of $\mathrm{Sn}$ is a well-documented function of pressure. ${ }^{24}$ In order to minimize $T_{c}$ shifts by remnant magnetic fields from the superconducting magnet a welldefined demagnetization procedure of the magnet was employed before each pressure determination. This resulted in a high reproducibility of the measured superconducting transition curves for each pressure. The inset of Fig. 1 shows the resistance data of the $\mathrm{Sn}$ gauge for selected pressures.

The Shubnikov-de Haas ( $\mathrm{SdH}$ ) signal, $\Delta \sigma=\sigma / \sigma_{b}-1$, is given by the relative conductance oscillations, with $\sigma_{b}$ the steady part of the conductivity. In general, a tensor inversion is necessary to obtain $\sigma$ from the longitudinal and transverse resistances. Since for the present material the Hall component of the resistivity tensor is negligible the $\mathrm{SdH}$ signal was calculated by $\Delta \sigma=R_{b} / R-1$, where the steady part of the resistance, $R_{b}$, was fitted by a low-power polynomial. Only from these kinds of data the $\mathrm{SdH}$ amplitudes were extracted by use of Fourier transformations.

\section{RESULTS AND DISCUSSION}

\section{A. Pressure-induced metal-insulator transition}

In this section, we first discuss the pressure dependence of the zero-field electrical-transport properties of 
$\beta^{\prime \prime}$-(BEDT-TTF) ${ }_{2} \mathrm{SF}_{5} \mathrm{CH}_{2} \mathrm{CF}_{2} \mathrm{SO}_{3}$. Figure 1 shows the interplane resistivity $\rho$ of sample 2 between 250 and $0.4 \mathrm{~K}$ measured during cooldown for different selected pressures. ${ }^{25}$ The absolute value of $\rho$ may be wrong by $30 \%$ due to the small and irregularly shaped crystal morphology. Nevertheless, for different crystals similar resistivities within these error bars could be determined. It is remarkable that for $\beta^{\prime \prime}$-(BEDT-TTF) ${ }_{2} \mathrm{SF}_{5} \mathrm{CH}_{2} \mathrm{CF}_{2} \mathrm{SO}_{3}$ the absolute value of $\rho$ $\approx 3 \mathrm{k} \Omega \mathrm{cm}$ at $p=0$ and room temperature is by about two orders of magnitude larger than the already large resistivity of other 2D organic metals. ${ }^{1,6}$ In spite of this extraordinary large resistivity a metallic temperature dependence of $\rho$ is observed over the whole temperature range. A maximum of $\rho(T)$, as previously reported by other groups, ${ }^{26,27}$ has never been observed in our samples. Therefore, an intrinsic origin of this maximum, which led to speculations of a possible density-wave state, ${ }^{26}$ can definitely be excluded.

At low temperatures (below about $20 \mathrm{~K}$ ) a quadratic temperature dependence of $\rho$ is found for all data shown in Fig. 1 (see also Fig. 5 below). This is usually taken as evidence for a well-behaved Fermi liquid where scattering is dominated by electron-electron interactions. However, although electronic correlations are certainly present in these lowdimensional metals, the wide temperature range over which the $T^{2}$ behavior can be found and the very large absolute value of the $T^{2}$ term is not explicable within the usual Fermiliquid theory. Indeed, other previously reported experimental observations suggest as well a failure of the semiclassical Boltzmann transport theory for $\beta^{\prime \prime}$-(BEDT-TTF) ${ }_{2} \mathrm{SF}_{5} \mathrm{CH}_{2} \mathrm{CF}_{2} \mathrm{SO}_{3} \cdot{ }^{28}$

To be more precise, a fit of the low-temperature resistivity by the function $\rho(T)=\rho_{0}+A T^{2}$ results in $A \approx 0.4 \Omega \mathrm{cm} /$ $\mathrm{K}^{2}$ for $p=0$. With increasing pressure, $A$ reduces unexpectedly fast to $A \approx 0.02 \Omega \mathrm{cm} / \mathrm{K}^{2}$ at $p=11.7 \mathrm{kbars}$. This shows clearly that the $T^{2}$ dependence of $\rho$ in organic chargetransfer salts cannot be understood by conventional electronelectron scattering. First, $A \approx 0.4 \Omega \mathrm{cm} / \mathrm{K}^{2}$ is about eight orders of magnitude larger than expected from the KadowakiWoods scaling ${ }^{29}$ for heavy-fermion compounds with comparable Sommerfeld coefficient $\gamma$, for which electronic correlations are certainly much more dominant than for organic metals. (Specific-heat data of the organic superconductor discussed here resulted in $\gamma \approx 19 \mathrm{~mJ} \mathrm{~mol}^{-1} \mathrm{~K}^{-2} \cdot{ }^{16}$ ) Second, $A$ does not scale with $m_{c}$ (see below). Usually, $A$ $\propto \gamma^{2} \propto m_{c}^{2}$ is observed for metals belonging to the same class of materials. ${ }^{29}$ Hence, a different mechanism has to be invoked to account for the $T^{2}$ dependence.

As seen in Fig. 1, the application of hydrostatic pressure considerably enhances the interlayer conductivity as usually observed for organic metals. This can qualitatively be understood by an increasing interlayer hopping integral $t_{\perp}$ caused by the larger pressure-induced overlap of the molecular orbitals. Nevertheless, $t_{\perp}$ still remains extremely small, namely, $t_{\perp}<1 \mu \mathrm{eV}$, since-as discussed in detail for $p=0$ (Ref. 28) - no indications for a 3D corrugated Fermi surface could be detected up to the highest pressures (see below). Noticeable is the very strong initial decrease of $\rho$ with pres-

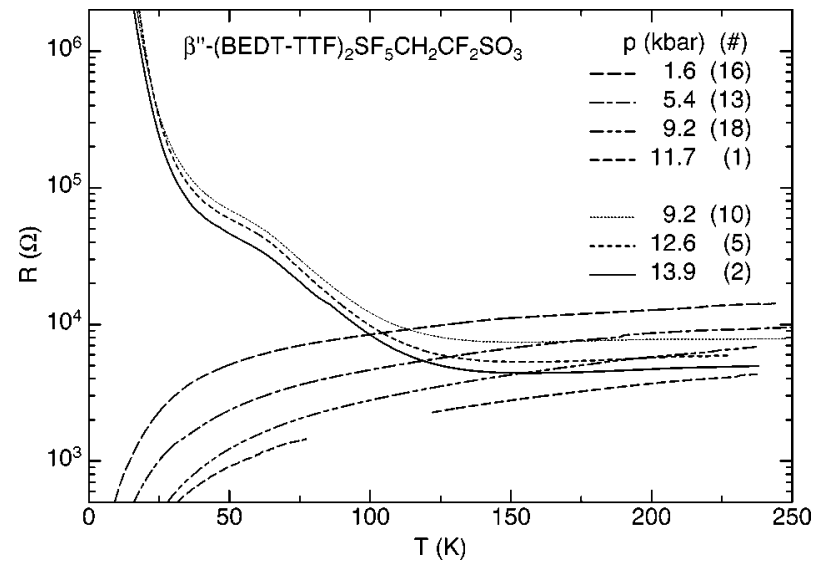

FIG. 2. Temperature dependence of the resistance of sample 2 for different selected pressures in a half-logarithmic scale. The numbers in brackets denote the chronological sequence of the measurements.

sure at room temperature. This might partially be caused by the usual pressure loss inside the pressure cell during cooling, i.e., the pressure acting on the sample decreases from an unknown strength at room temperature (usually 2-3 kbars larger than at low temperatures) to the values stated in Fig. 1. Another possibility might be a stronger thermal expansion of the samples at $p=0$.

A rather unexpected feature is the observation of insulating behavior of $\beta^{\prime \prime}$-(BEDT-TTF) ${ }_{2} \mathrm{SF}_{5} \mathrm{CH}_{2} \mathrm{CF}_{2} \mathrm{SO}_{3}$ at higher pressures. In Fig. 2 two principally different temperature dependences of $R(T)$ can be realized. Below a certain pressure, the already discussed metallic and eventually superconducting behavior occurs. For higher pressures above about 12 kbars, the material shows a metal-like resistance only for $T$ $\gtrsim 153 \mathrm{~K}$, whereas towards lower temperatures insulating behavior arises. Upon reducing the pressure from above 12 kbars again, the metallic state can be reached only for pressures below about 9 kbars for sample 2 (below 7.4 kbars for sample 1).

In the insulating state the overall temperature dependence of $R(T)$ changes merely with small relative reductions towards higher pressures. The resistance shows a semiconducting temperature dependence which can be described by an activated behavior at low temperatures, i.e., $R(T)$ $\propto \exp \left[E_{g} /\left(2 k_{B} T\right)\right]$. In an Arrhenius plot (Fig. 3) the approximately linear behavior of the data below about $30 \mathrm{~K}$ can be seen. An energy gap of $E_{g} \approx 17 \mathrm{meV}$ can be inferred common to all pressures in the insulating state. The origin of the structure in $R(T)$ around $70 \mathrm{~K}$ is unclear, but might be related to a contribution coming from the $1 \mathrm{D}$ bands.

In Fig. 4 the resistances of sample 2 at $77 \mathrm{~K}$ and at room temperature (before loading the probe into the cryostat) are plotted as a function of pressure. The numbers next to the $77-\mathrm{K}$ data points denote the temporal sequence of the measurements (see also Fig. 2). At room temperature, a small increase of the resistance at $p>11.7$ kbars hints towards the insulating state. The data at $77 \mathrm{~K}$ allow more clearly to separate the metallic and the insulating regions. Between 9 and 12 kbars hysteretic behavior is observed. Depending on whether the pressure is increased or released (arrows in Fig. 4) either the metallic or the insulating state occurs. This hys- 


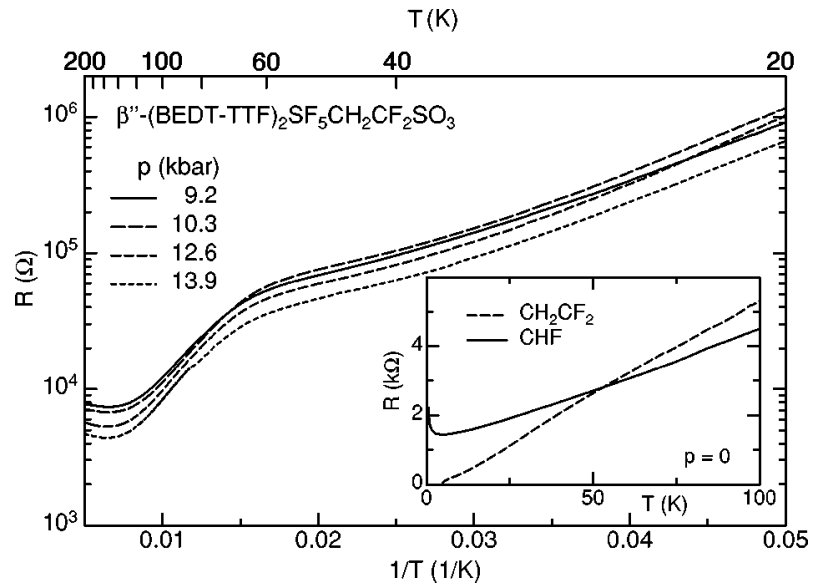

FIG. 3. Arrhenius plot of the resistance of sample 2 in the insulating state for selected pressures reflecting the activated behavior at low $T$. The inset shows the temperature dependences of the resistances of $\beta^{\prime \prime}-(\mathrm{BEDT}-\mathrm{TTF})_{2} \mathrm{SF}_{5} X \mathrm{SO}_{3}$ for $X=\mathrm{CH}_{2} \mathrm{CF}_{2}$ and $X$ $=\mathrm{CHF}$ at $p=0$.

teresis was traversed twice for sample 2, proving that switching between both states is reversible. When increasing the pressure for the second time the insulating state appeared already slightly above 10 kbars (point 19 in Fig. 4). However, it cannot be excluded that a somewhat higher pressure had been applied briefly before fixing the piston at room temperature. Subsequently, the pressure was released far enough to restore the metallic state and then again carefully increased to about 10 kbars (point 20) to reconfirm the hysteresis.

This hysteretic behavior is a clear manifestation for a first-order phase transition which hints towards a structural transition of the material. If true this structural modification must be of subtle nature since no major lattice changes could be detected in first neutron-scattering experiments under pressure. ${ }^{30}$ Further studies are necessary to determine the detailed structure under pressure.

It is known that the charge-transfer salts of the family $\beta^{\prime \prime}$-(BEDT-TTF $)_{2} \mathrm{SF}_{5} R \mathrm{SO}_{3}$ show drastically different

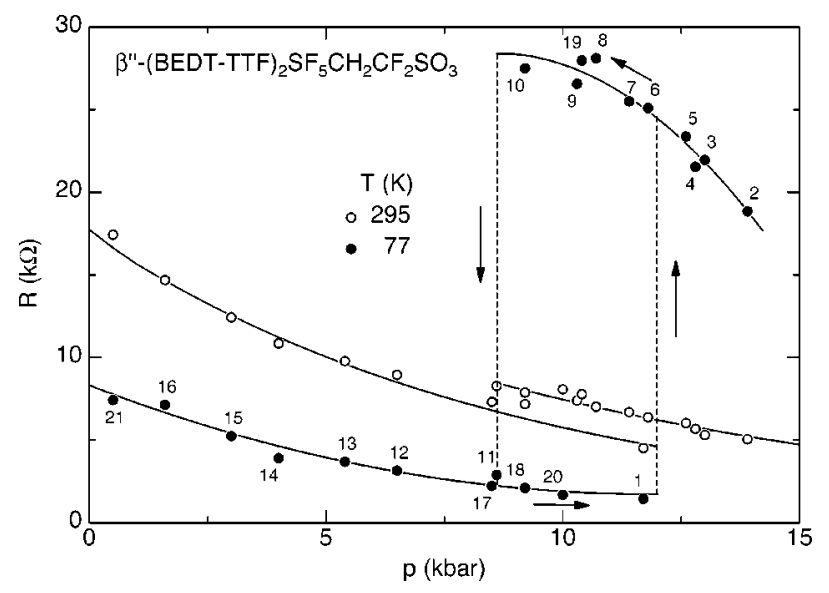

FIG. 4. Pressure dependence of the resistance at 295 and $77 \mathrm{~K}$ for sample 2. The numbers denote the temporal sequence of the measurements. The lines are guides for the eye. ground states when changing the central part $R$ of the anion. ${ }^{19,20}$ Except for $R=\mathrm{CH}_{2} \mathrm{CF}_{2}$, discussed here, all other known isostructural compounds with modified $R$ are insulating already at room temperature $\left(R=\mathrm{CH}_{2}\right)$ or become insulating towards lower temperatures $\left(R=\mathrm{CHFCF}_{2}\right.$ below about $175 \mathrm{~K}$ and $R=\mathrm{CHF}$ below about $6 \mathrm{~K}$ ). The temperature dependence of the resistance for the latter material is shown in the inset of Fig. 3. Starting at high $T$ with a metallic behavior, similar to the superconducting compound, at the lowest temperatures a semiconducting resistance appears that can be described by an activated dependence with a very small energy gap of about $40 \mu \mathrm{eV}$. Up to date, it is unclear why these subtle changes in the anion layer, i.e., in regions which are not involved in the charge transport, lead to such drastically different ground states. Extended-Hückel bandstructure calculations suggest metallic behavior for all isostructural compounds.

A possible reason for the insulating behavior might be a charge disproportionation within the anion layer. The large difference in electronegativity of the anion constituents could lead to a charge-ordered state for the itinerant electrons in the donor layer. Experimentally, for $R=\mathrm{CH}_{2}$, an insulating material, a charge distribution of +0.4 and +0.6 was found for the BEDT-TTF molecules inside a unit cell. ${ }^{20}$ As mentioned, the theoretical study of the quarter-filled extended Hubbard model on a square lattice (relevant for the $\beta^{\prime \prime}$ structure) predicts a charge-ordered insulator at $T=0$ for a large intersite Coulomb repulsion $V .^{21}$ Changing the anion as well as pressure might sensitively increase $V$ leading to the observed behavior. However, the first-order nature of the transition is not in favor for a solely electronic origin of the metalinsulator transition.

\section{B. Electronic properties in the metallic state}

In the following, we discuss how the superconducting transition, the Fermi surface, and other electronic bandstructure properties change with pressure in the metallic state. As mentioned, previous experiments by use of a gas pressure cell up to about 2.3 kbars showed for $\beta^{\prime \prime}$-(BEDT-TTF) ${ }_{2} \mathrm{SF}_{5} \mathrm{CH}_{2} \mathrm{CF}_{2} \mathrm{SO}_{3}$ an approximately linear decrease of the superconducting transition temperature with a slope of $d T_{c} / d p=-1.34 \mathrm{~K} / \mathrm{kbar}^{4}$ Along these lines, we find a strong shift of the resistive-transition curves towards lower temperatures with increasing pressure (Fig. 5). By using the resistive midpoint as the criterion for $T_{c}$, we find an initial pressure-induced $T_{c}$ reduction fully in line with the above-stated slope [dashed line in Fig. 6(d)]. At higher pressures (above about $3 \mathrm{kbars}$ ) $T_{c}$ decreases less quickly. In fact, for all pressures up to $11.7 \mathrm{kbars}$, where a metallic resistance was observed, incipient superconductivity could be detected (inset of Fig. 5). In Fig. 6(d) this is visualized by triangles reflecting the fact that only onsets, i.e., less than $50 \%$, of the superconducting transition could be observed down to $0.4 \mathrm{~K}$. This result seems to indicate that for the present material superconductivity is omnipresent in the metallic state and we are dealing with a superconductor-insulator transition.

The ability to tune $T_{c}$ so easily with pressure in connection with the constant high sample quality of the present 


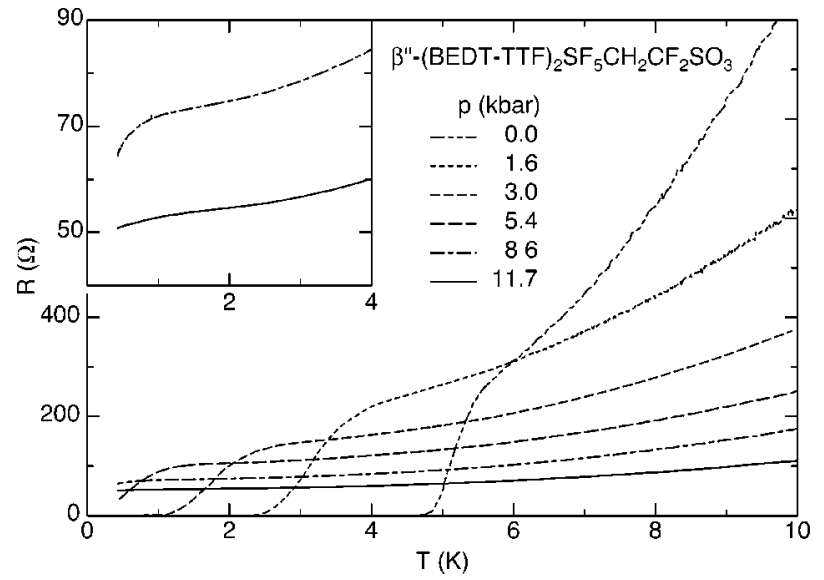

FIG. 5. Low-temperature part of the resistance of sample 2 for selected pressures. The inset reflects in an enlarged scale that superconducting fluctuations are present up to the highest pressures ( 8.6 and $11.7 \mathrm{kbars})$ in the metallic state.

organic superconductor allows one to investigate the relationship between the electronic band-structure properties and superconductivity over a broad parameter range. Thereby, the possibility to rotate the sample around two axes in our experimental setup permits a full determination of the electronic band-structure parameters for all pressures.

From $\mathrm{SdH}$, de Haas-van Alphen (dHvA), and angulardependent magnetoresistance (AMRO) data at $p=0$ it is known that $\beta^{\prime \prime}-(\mathrm{BEDT}-\mathrm{TTF})_{2} \mathrm{SF}_{5} \mathrm{CH}_{2} \mathrm{CF}_{2} \mathrm{SO}_{3}$ has a single perfectly $2 \mathrm{D}$ Fermi surface that occupies about $5 \%$ of the $2 \mathrm{D}$ Brillouin zone resulting in a quantum-oscillation frequency of $F_{0}=199$ (1) T. The effective cyclotron mass for this orbit is $m_{c}=2.0(1) m_{e}$, where $m_{e}$ is the free-electron mass and $g m_{c} / m_{e}=3.92(1)$ is determined for the electron $g$ factor times $m_{c} \cdot{ }^{31-33}$ Although the Fermi-surface topology is qualitatively in line with band-structure calculations, an (at least

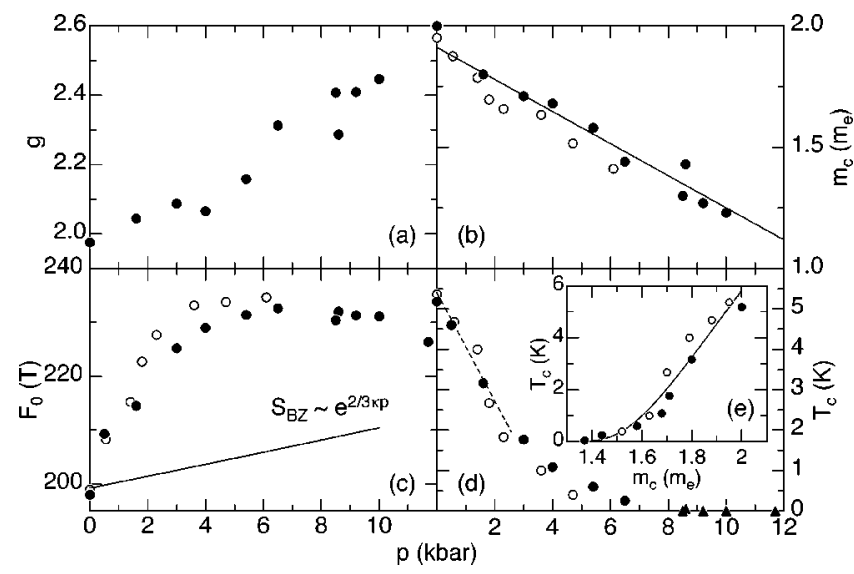

FIG. 6. Pressure dependences of (a) the electron $g$ factor, (b) the effective cyclotron mass $m_{c}$, (c) the $\mathrm{SdH}$ oscillation frequency at $\Theta=0$, and (d) the superconducting transition temperature $T_{c}$. For the different lines see text. Inset (e) shows $T_{c}$ as a function of $m_{c}$ with a fit (solid line) according to the modified McMillan equation (5) as described in the text. Open circles are for sample 1 and closed circles for sample 2 .

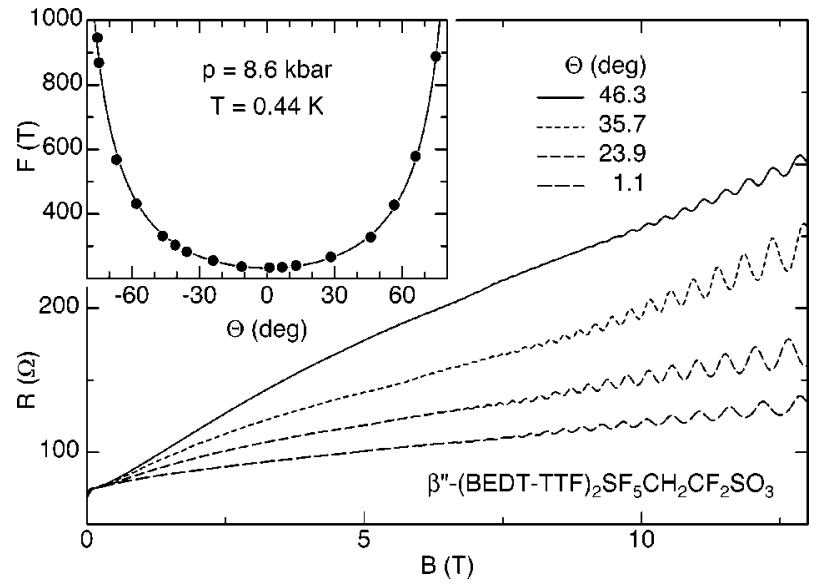

FIG. 7. Shubnikov-de Haas oscillations at $p=8.6 \mathrm{kbars}$ and $T$ $=0.44 \mathrm{~K}$ for different angles. The inset shows the angular dependence of the oscillation frequency. The solid line reflects the expected $2 \mathrm{D} 1 / \cos \Theta$ dependence.

for organic charge-transfer salts) unusual quantitative discrepancy exists. ${ }^{31}$ Experimentally the Fermi surface is about three times smaller than predicted, namely, the ellipsoidal length of the semiminor axis, i.e., the width approximately along the $k_{b}$ direction, is about one-third the calculated value. $^{32}$ Furthermore, band-structure calculation predicts open 1D bands which could not be verified experimentally (e.g. by a magnetic-breakdown orbit). Anyway, the detectable quantum-oscillation signal is simple with only one fundamental frequency and in almost perfect agreement with the behavior expected for a 2D metal with fixed chemical potential. $^{33}$

Under pressure we could observe $\mathrm{SdH}$ oscillations up to the highest pressures for which the samples remained metallic. As an example, Fig. 7 shows the magnetoresistance at $p=8.6 \mathrm{kbars}$ and $T=0.44 \mathrm{~K}$ for different angles. Clear oscillations could be detected starting at about $4 \mathrm{~T}$ for all pressures. This shows that the crystal quality did not deteriorate under pressure due to, e.g., pressure inhomogeneities. Indeed, the Dingle temperatures for different pressures stay approximately constant and lie within $T_{D}=(1.2 \pm 0.2) \mathrm{K}$. Thereby, $T_{D}=\hbar / 2 \pi k_{B} \tau$ is determined from the fielddependent damping of the $2 \mathrm{D} \mathrm{SdH}$ signal, with $k_{B}$ the Boltzmann constant and $\tau$ the quasiparticle scattering time. ${ }^{34}$

For all pressures the expected 2D angular dependence of the $\mathrm{SdH}$ oscillation frequency $F=F_{0} / \cos \Theta$ was found (inset of Fig. 7). Thereby, the fundamental frequency $F_{0}$ for $\Theta$ $=0$, i.e., the extremal cross section of the Fermi surface, $a_{F}=2 \pi e F / \hbar$, shows a nonmonotonic pressure dependence. Up to 4 kbars, $F_{0}$ increases rapidly from 199(1) T at ambient pressure to about $233 \mathrm{~T}$. For higher pressures, $F_{0}$ first remains constant before the Fermi-surface area decreases again reaching $226(1) \mathrm{T}$ at $p=11.7 \mathrm{kbars}$ close to the insulating state [see Fig. 6(c)].

An increase of the Fermi-surface area under pressure is qualitatively expected since the unit-cell volume in real space gets reduced, i.e., the Brillouin zone in $k$ space increases. More precisely, for a single-band metal $F_{0}$ would be directly proportional to the $2 \mathrm{D}$ Brillouin-zone area. In order 
to estimate the relative change of the Brillouin-zone area $S_{B Z}$, we used a typical compressibility for organic metals of $\kappa=(122 \mathrm{kbars})^{-1} \cdot{ }^{35}$ By assuming an isotropic and pressureindependent compressibility $S_{B Z} \propto \exp \left(\frac{2}{3} \kappa p\right)$ would be expected. This would result in the increase of $F_{0}$ as plotted as the solid line in Fig. 6(c). It is evident that the estimated increase (about $2.3 \%$ at 4 kbars) is much less than that experimentally observed. One could argue that the assumed isotropic compressibility might not be justified. Indeed, for $\beta$-(BEDT-TTF $)_{2} \mathrm{I}_{3}$ x-ray measurements under pressure showed a factor of 2 larger in-plane compressibility than that perpendicular to the planes. ${ }^{36}$ However, even this anisotropy of the compressibility could not explain the large increase of the Fermi-surface area.

For $\beta^{\prime \prime}$-(BEDT-TTF) ${ }_{2} \mathrm{SF}_{5} \mathrm{CH}_{2} \mathrm{CF}_{2} \mathrm{SO}_{3}$, in addition to the closed hole orbit responsible for the $\mathrm{SdH}$ signal open bands are predicted by band-structure calculations. ${ }^{31}$ Therefore, the area of the closed orbit crossing the Brillouin-zone border is not necessarily proportional to the Brillouin-zone area. An analogous behavior has been found for $\kappa$-(BEDT-TTF $)_{2} \mathrm{Cu}(\mathrm{NCS})_{2}$ which has a topologically similar Fermi surface. ${ }^{9,13}$ For this material, the so-called $\alpha$-orbit area showed an increase larger than expected. At higher fields, in this metal magnetic breakdown allows the charge carriers to traverse the $1 \mathrm{D}$ bands in the so-called $\beta$ orbit. As a result of the band filling the $\beta$-orbit area agrees exactly with the Brillouin-zone area which indeed was confirmed to be valid also under pressure. ${ }^{9,13}$

It remains however an open question why the Fermisurface area decreases again for higher pressures close to the insulating state. Detailed information on the lattice parameters under pressure would be helpful to decide whether this is caused by bare structural changes or whether the effect has an electronic origin.

In this context it was of interest to follow the detailed Fermi-surface topology under pressure. For this purpose we utilized AMRO measurements for all pressures in the metallic state (see data in Ref. 37). By measuring the angular positions where pronounced maxima in the AMRO signal occur (the so-called Yamaji angles), ${ }^{38,39}$ the in-plane Fermi surface can be mapped out in detail. No topological change of the strongly elongated ellipsoidal Fermi surface (axis ratio 1:9) $)^{32}$ was found, i.e., the AMRO maxima for all azimuthal angles remained at almost fixed positions for all pressures. An observed slight change of the in-plane Fermi wave vector is in line with the pressure dependence of $F$ discussed above.

As mentioned above, at $p=0$ any proof for a 3D corrugated Fermi surface is absent. ${ }^{28}$ Especially, no beating of the $\mathrm{SdH}$ signal and no peak at $\Theta=90^{\circ}$ exist. This means that $\beta^{\prime \prime}$-(BEDT-TTF) ${ }_{2} \mathrm{SF}_{5} \mathrm{CH}_{2} \mathrm{CF}_{2} \mathrm{SO}_{3}$ is one of the best realizations of a perfect $2 \mathrm{D}$ metal (cf. also the ideal $2 \mathrm{D}$ dHvA signal). ${ }^{33}$ With increasing pressure, the Fermi surface should eventually become three dimensional and consequently the mentioned features should emerge. Nevertheless, up to 11.7 kbars no indication for a 3D Fermi surface can be found. Neither beats of the SdH signal nor a peak at $90^{\circ}$ appeared.

Information on further band-structure parameters can be obtained from the temperature and angular dependences of the SdH signal. The amplitude of the fundamental SdH oscillation for a $2 \mathrm{D}$ metal is given by

$$
A_{1} \propto F B R_{D} R_{T} R_{S},
$$

with the usual Dingle $\left(R_{D}\right)$, temperature $\left(R_{T}\right)$, and spinsplitting $\left(R_{S}\right)$ damping factors. ${ }^{34}$ After calculating the $\mathrm{SdH}$ signal $\Delta \sigma / \sigma$, i.e., the relative conductance oscillations, ${ }^{40}$ we extracted the fundamental amplitude from Fourier transformations of the data. From the temperature dependence of $A_{1}$ the pressure dependence of the effective cyclotron mass $m_{c}$, shown in Fig. 6(b), was extracted (all data for $\Theta=0$ ). Starting from $m_{c}=2.0(1) m_{e}$ at $p=0$ an approximately linear decrease of $m_{c}$ [solid line in Fig. 6(b)] is found. At $p$ $=10 \mathrm{kbars}, m_{c}=1.23(10) m_{e}$ is close to the bare band mass $m_{b}=1.07 m_{e}$ predicted by band-structure calculations. ${ }^{31}$ The effective cyclotron mass is equal to the band mass enhanced by many-body effects such as electron-phonon and electronelectron interactions. This is usually described by ${ }^{34}$

$$
m_{c}=m_{b}\left(1+\lambda_{e p}\right)\left(1+\lambda_{e e}\right) .
$$

For the free-electron-gas model, $m_{b}$ is not a function of pressure. Therefore, the observed strong decrease of $m_{c}$ indicates that many-body interactions become weaker at higher pressures. It is, however, not possible from the present experiments to disentangle the different contributions of the two enhancement factors.

Some further insight in the relevance of electronic correlations can be gained from the determination of the electron $g$ factor occurring in the spin-splitting damping factor, which for the fundamental amplitude is given by

$$
R_{S}=\cos \left(\frac{\pi g m_{c}}{2 m_{e}}\right) .
$$

This factor becomes zero at those angles for which $g m_{c} / m_{e}=2 n-1$, where $n$ is an integer. Since $m_{c}$ increases with angle proportional to $1 / \cos \Theta$, from the angular positions for which the fundamental $\mathrm{SdH}$ amplitude vanishes fairly exact values of $g m_{c}$ can be determined. This results in $g m_{c} / m_{e}=3.92(2)$ at $p=0$ which yields $g=1.96$ for $m_{c}$ $=2.0$. A value of $g \approx 2$ is consistent with the SommerfeldWilson ratio as discussed in Ref. 41. It further may be compared to the electron-spin-resonance (ESR) value $g_{E S R}$ $=2.01 .{ }^{23}$ In spite of this good agreement, $g$ and $g_{E S R}$ are not necessarily identical since $g$ is renormalized by many-body interactions whereas $g_{E S R}$ is not, i.e., ${ }^{34}$

$$
g=\frac{g_{E S R}}{\left(1+\lambda_{e p}\right)\left(1+\lambda_{e e}^{\prime}\right)},
$$

where the parameter for electron-electron interaction $\lambda_{e e}^{\prime}$ is different from $\lambda_{e e}$ renormalizing $m_{c}$. The principal existence of electronic correlations in organic metals has been proven by $\mathrm{dHvA}$ and $\mathrm{SdH}$ studies as well as by many other experiments. ${ }^{1}$ According to Kohn's theorem, the effective mass extracted from magneto-optical cyclotron-resonance 


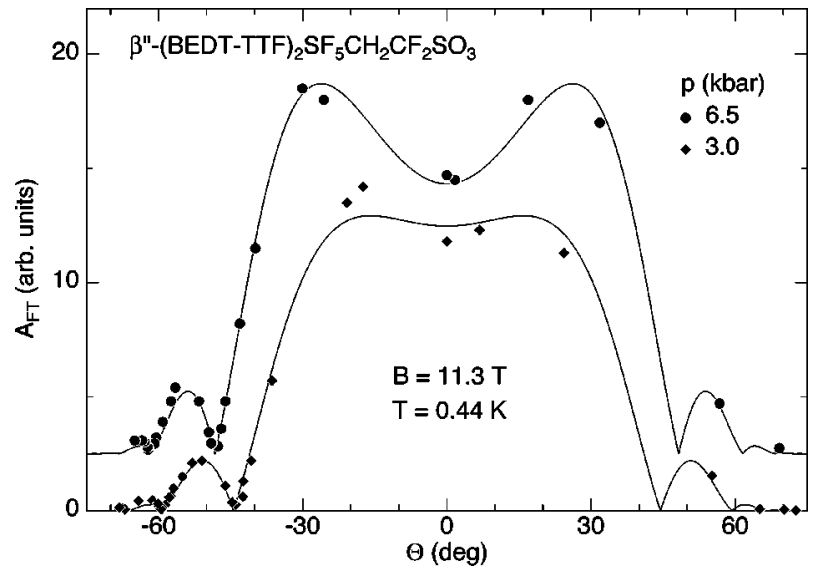

FIG. 8. Angular dependence of the fundamental SdH oscillation amplitude for two different pressures. The data at $6.5 \mathrm{kbars}$ are shifted upward by 2.5 . The solid lines are fits according to Eq. (1) from which rather exact values for $g m_{c}$ can be obtained.

data is assumed to be not renormalized by $\left(1+\lambda_{e e}\right) .{ }^{42}$ However, in contradiction to this theoretical prediction a cyclotron-resonance mass of 2.26(3) $m_{e}$ has recently been reported for the present material. ${ }^{43}$ This shows that electronic correlations are present and that they may influence magneto-optical masses. Indeed, as was pointed out recently, for these layered metals, one should not expect Kohn's theorem to be applicable. ${ }^{44}$

It is therefore reasonable to assume that the agreement between $g$ and $g_{E S R}$ found at $p=0$ is accidental. By fitting Eq. (1) to the measured angular dependence of $A_{1}$ (Fig. 8) we can extract, besides a simple scaling factor and $T_{D}$ (which comes out somewhat larger than the values determined from the field dependence of $\left.A_{1}\right), g m_{c}$ for different pressures. With the measured data for $m_{c}$ [Fig. 6(b)] we finally obtain the pressure dependence of $g$ [Fig. 6(a)]. Starting from the nearly free-electron value $1.96, g$ increases approximately linearly up to $g=2.45$ at $p=10$ kbars. This clearly shows the existence and importance of electronic correlations in $\beta^{\prime \prime}$-(BEDT-TTF) ${ }_{2} \mathrm{SF}_{5} \mathrm{CH}_{2} \mathrm{CF}_{2} \mathrm{SO}_{3}$, although absolute numbers for $\lambda_{e e}$ and $\lambda_{e e}^{\prime}$ may not be deduced from the present dataset.

Now that the pressure dependences of the relevant bandstructure parameters are available a quantitative description of $T_{c}$ as a function of $p$ may be tried by use of the modified McMillan equation ${ }^{45}$

$$
T_{c}=\frac{\langle\omega\rangle}{1.2} \exp \left(-\frac{1.04(1+\lambda)}{\lambda-\mu^{*}(1+0.62 \lambda)}\right)
$$

Usually, $\langle\omega\rangle$ represents an average phonon frequency, $\lambda$ $=\lambda_{e p}$, and the Coulomb pseudopotential is set to $\mu^{*}=0.1$. By assuming $\lambda=m_{c} / m_{b}-1$, i.e., setting $\lambda_{e e}=0,{ }^{46}$ we obtain a good description of the data as shown in Fig. 6(e). The fit parameters $\langle\omega\rangle / 1.2=97(13) \mathrm{K}$ and $m_{b}=1.03(2) m_{e}$ are reasonable in spite of the simple formula and the crude approximations. As mentioned, band-structure calculations predict $m_{b}=1.07$ which is in very good agreement with the fit

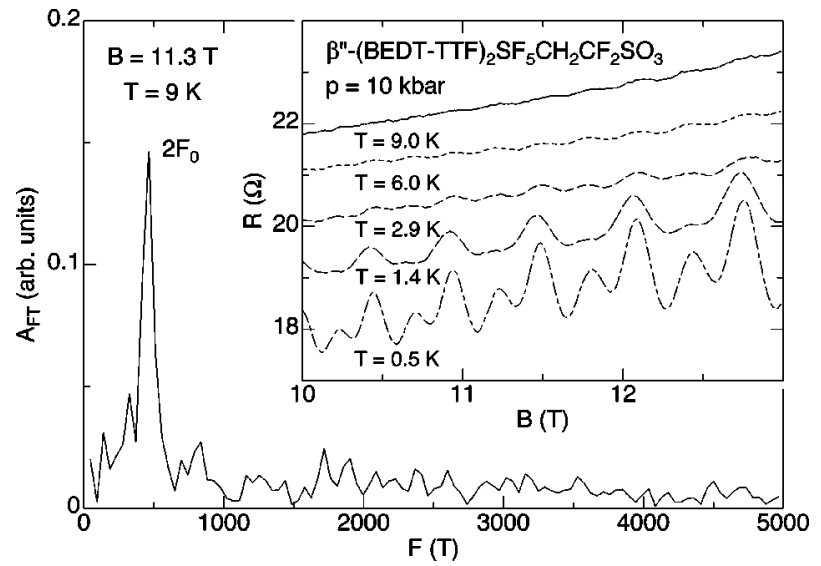

FIG. 9. Fourier spectrum of the $\mathrm{SdH}$ signal for sample 2 at 10 kbars and $9 \mathrm{~K}$. No resolvable signal at $F_{0}$, but a sharp peak at $2 F_{0}=460 \mathrm{~T}$ is visible. The inset shows the magnetoresistance data for different temperatures between 0.5 and $9 \mathrm{~K}$. The data are shifted consecutively for better visibility.

value. ${ }^{31}$ For $p=0$, the fit gives a coupling constant of $\lambda$ $=0.94(4)$. Specific-heat data result in a Debye temperature $\Theta_{D}=220(10) \mathrm{K}$ and in $\lambda=1.1(1)$ obtained from the jump at $T_{c}$ by use of a phenomenological theory. ${ }^{16,47}$ The coupling constants extracted from these independent measurements are in excellent agreement. Since $\Theta_{D}$ gives an upper limit for the relevant phonon frequencies, the fit value $\langle\omega\rangle / 1.2$ $=97(13) \mathrm{K}$ appears to be realistic. ${ }^{48}$ Consequently, the modified McMillan equation provides a physically appropriate description of $T_{c}(p)$.

\section{Electronic properties close to the insulating state}

As the final point we discuss the electronic properties close to the metal-insulator transition. Although this transition seems to be caused by small structural changes in the unit cell, it might be favored or even induced by electronic modifications. In this context the plateau above 4 kbars and the unexpected decrease of the Fermi-surface area close to the insulating state should be remembered [Fig. 6(c)].

Another feature indicates that the band structure changes already below the transition. When following the $\mathrm{SdH}$ signal at $p=10$ kbars, i.e., within the hysteretic region, oscillations with twice the fundamental frequency could be resolved clearly up to unusually high temperatures. The inset of Fig. 9 shows the measured field dependence of $R$ at $\Theta=0$. For this pressure a spin-splitting zero occurs close to this angle $\left(g m_{c} / m_{e} \approx 3\right)$. Therefore, at $T=0.5 \mathrm{~K}$ a strong second harmonic is visible besides the fundamental frequency $F_{0}$ $=230 \mathrm{~T}$. Towards higher $T$ the fundamental vanishes rapidly, whereas an oscillation with $2 F$ remains as visualized by the Fourier transformation of the data at $T=9 \mathrm{~K}$ (Fig. 9). The amplitude $A_{2}$ of this oscillation is much too large for being simply the second harmonic of $F$.

This can be seen in a more quantitative way when $A_{2}$ is plotted as a function of temperature (Fig. 10). Only below about $1 \mathrm{~K}$ the data can be described by using an effective mass of $m_{c}=1.23 m_{e}$ (dashed line in Fig. 10) as extracted 


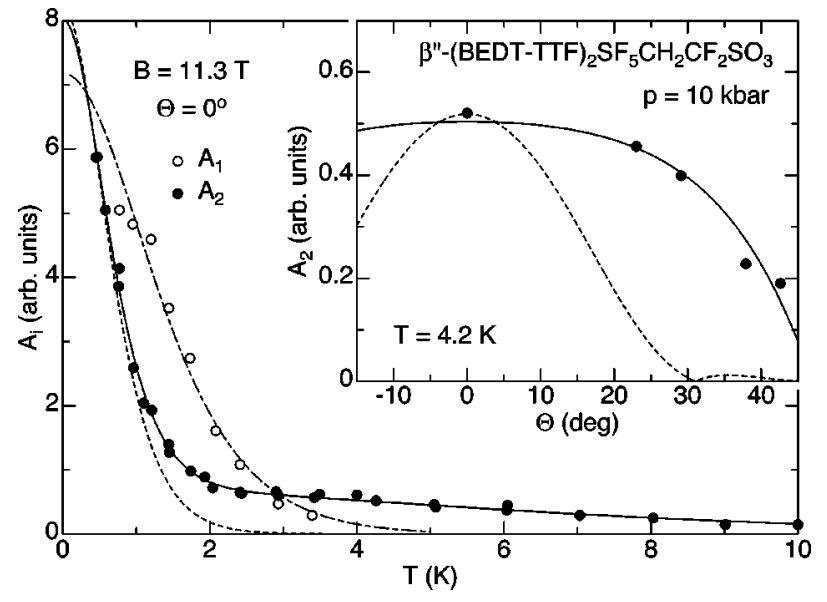

FIG. 10. Temperature dependence of the fundamental amplitude $A_{1}$ and the second-harmonic $\mathrm{SdH}$ amplitude $A_{2}$ at $10 \mathrm{kbars}$. Below $1 \mathrm{~K}, A_{2}$ can be described by use of the effective mass $m_{c}$ $=1.23 m_{e}$ (dashed line) as obtained from the temperature dependence of the fundamental signal $A_{1}$ (dash-dotted line). Only by assuming additional charge carriers with an effective mass of $0.26 m_{e}$ all data of $A_{2}$ up to the highest temperature can be described (solid line). The inset shows the angular dependence of $A_{2}$ at $T$ $=4.2 \mathrm{~K}$. The curves are described in the text.

from the temperature dependence of the fundamental $A_{1}$ (dash-dotted line). It is evident that an additional contribution takes part in the oscillating signal becoming visible towards higher temperatures. A reasonable description of $A_{2}(T)$ is obtained by assuming two $\mathrm{SdH}$ signals with the same frequency but different effective masses (solid line in Fig. 10). Thereby, the resulting larger effective mass of $m_{c}$ $=1.23(3) m_{e}$ (for a second harmonic) agrees excellently with the fundamental value stated above, whereas the rather small mass of $m_{c 2}=0.26$ (3) $m_{e}$ (for the additional fundamental signal) may be ascribed to additional charge carriers.

In order to check further that the $\mathrm{SdH}$ signal with $2 F_{0}$ at higher $T$ is indeed not caused by the charge carriers on the known hole orbit we investigated the angular dependence of $A_{2}$ at $4.2 \mathrm{~K}$ (inset of Fig. 10). In case these charge carriers would be responsible for extraordinary large oscillations with $2 F_{0}$ the amplitude $A_{2}$ would vanish at those angles $\Theta$ where the second-harmonic spin-splitting factor $R_{S 2}$ $=\cos \left(\pi g m_{c} / m_{e}\right)$ becomes zero. With $g m_{c} / m_{e}=3.01$ extracted from the angular dependence of the fundamental and $m_{c} \propto 1 / \cos \Theta$ the dashed line in the inset of Fig. 10 results. This line, obtained with an unphysical large prefactor, deviates significantly from the measured data. Alternatively, for a new fundamental signal originating from a $2 \mathrm{D}$ orbit with $g m_{c 2} / m_{e}=0.68(2)$, i.e., assuming an electron $g$ factor of $g$ $\approx 2.6(4)$, Eq. (1) leads to the good description of the data shown by the solid line in the inset of Fig. 10. This $g$ factor agrees well with $g \approx 2.45(5)$ obtained for the charge carriers on the hole orbit.

Consequently, our results give good support for a reconstructed band structure already below the insulating state. The detailed modification of the band structure and why it occurs is, however, unclear. The additional oscillating signal might either be caused by a new Fermi-surface orbit or origi-

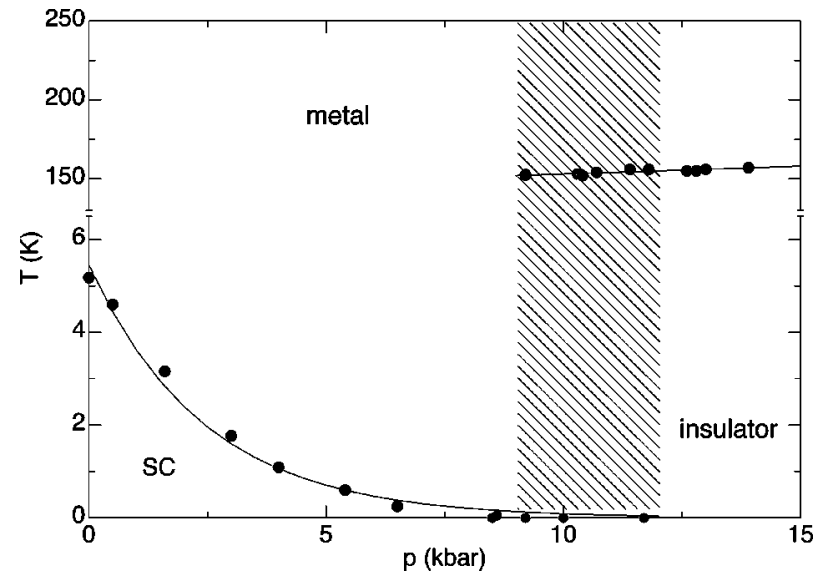

FIG. 11 . $P-T$ phase diagram of $\beta^{\prime \prime}$-(BEDT-TTF) ${ }_{2} \mathrm{SF}_{5} \mathrm{CH}_{2} \mathrm{CF}_{2} \mathrm{SO}_{3}$. At pressures below about 12 kbars superconductivity (SC) is observed. Above $\sim 9$ kbars, insulating behavior occurs below about $153 \mathrm{~K}$. Between 9 and $12 \mathrm{kbars}$ both the metallic and the insulating state can be stabilized (hatched area). The lines are guides to the eye.

nating from a quantum interference effect. The latter occurs between carriers traveling along open orbits which cross or come close to each other in at least two positions in $k$ space. ${ }^{39,49}$ It might be that pressure reduces the distance between the predicted 1D bands ${ }^{31}$ which then could give rise to the observed feature. Measurements of the thermodynamic dHvA effect under pressure would be helpful to distinguish between the two possibilities. It remains to be clarified why the observed band-structure reconstruction precedes the appearance of the insulating state and how both influence each other.

\section{CONCLUSION}

The $P-T$ phase diagram of the organic superconductor $\beta^{\prime \prime}$-(BEDT-TTF) ${ }_{2} \mathrm{SF}_{5} \mathrm{CH}_{2} \mathrm{CF}_{2} \mathrm{SO}_{3}$ summarizing our results is shown in Fig. 11. A pressure-induced first-order phase transition between a metallic and an insulating state is observed between 9 and 12 kbars. At high pressure, on the insulating side, a smooth crossover from a metallic to an insulating behavior appears at about $153 \mathrm{~K}$. At low pressure, on the metallic side, superconductivity or at least the onset of superconductivity occurs. On the metallic side close to the transition clear changes of the electronic band-structure were detected. The phase diagram is quite different to what was expected from the quarter-filled Hubbard model. ${ }^{21}$ For that one would expect that $T_{c}$ and $m_{c}$ should increase when approaching the insulating phase. Hence, a structural phase transition changing the electronic bandwidth seems to be a plausible origin for the observed behavior although no proof can be given so far.

On the metallic side the main features of the Fermisurface topology remain unchanged with pressure. The Fermi-surface area of the 2D charge carriers first increases much faster than explained simply by the lattice compression. Close to the insulating state it decreases again. Contrary 
to this nonmonotonic behavior, the effective mass decreases approximately linearly with $p$ and the electron $g$ factor increases. This reflects the existence of strong many-body interactions and their large pressure sensitivity. The pressureinduced reduction of $T_{c}$ can be well described by use of the modified McMillan equation assuming a coupling strength $\lambda$ which is proportional to the measured cyclotron mass $m_{c}$. We find realistic physical parameters and good agreement with $\lambda$ extracted from specific-heat data for $p=0 .{ }^{16}$

\section{ACKNOWLEDGMENTS}

We thank R. H. McKenzie and J. Merino for helpful discussions. Financial support by the Deutsche Forschungsgemeinschaft is gratefully acknowledged. Work at Argonne National Laboratory was supported by the U.S. Department of Energy (Grant No. W-31-109-ENG-38). Work at Portland State University was supported by NSF (Grant No. Che9904316).
${ }^{1}$ T. Ishiguro, K. Yamaji, and G. Saito, Organic Superconductors, 2nd ed. (Springer, Berlin, 1998).

${ }^{2}$ D. Jérome, A. Mazaud, M. Ribault, and K. Bechgaard, J. Physique Lett. 41, L95 (1980).

${ }^{3}$ J.E. Schirber, E.L. Venturini, A.M. Kini, H.H. Wang, J.R. Witworth, and J.M. Williams, Physica C 152, 157 (1988).

${ }^{4}$ S. Sadewasser, C. Looney, J.S. Schilling, J.A. Schlueter, J.M. Williams, P.G. Nixon, R.W. Winter, and G.L. Gard, Solid State Commun. 104, 571 (1997).

${ }^{5}$ M. Tinkham, Introduction to Superconductivity (McGraw-Hill, New York, 1996).

${ }^{6} \mathrm{~J}$. Wosnitza, in Studies of High-Temperature Superconductors, edited by A.V. Narlikar (Nova Science, Huntington, 2000), Vol. 34; J. Wosnitza, Curr. Opin. Solid State Mater. Sci. 5, 131 (2001).

${ }^{7}$ J. Singleton, Rep. Prog. Phys. 63, 1111 (2000).

${ }^{8}$ S.J. Klepper, J.S. Brooks, X. Chen, I. Braderic, M. Tokumoto, N. Kinoshita, Y. Tanaka, and C.C. Agosta, Phys. Rev. B 48, 9913 (1993).

${ }^{9}$ J. Caulfield, W. Lubczynski, F.L. Pratt, J. Singleton, D.Y.K. Ko, W. Hayes, M. Kurmoo, and P. Day, J. Phys.: Condens. Matter 6, 2911 (1994).

${ }^{10}$ J.S. Brooks, X. Chen, S.J. Klepper, S. Valfells, G.J. Athas, Y. Tanaka, T. Kinoshita, N. Kinoshita, M. Tokumoto, H. Anzai, and C.C. Agosta, Phys. Rev. B 52, 14457 (1995).

${ }^{11}$ P. Auban-Senzier, A. Audouard, V.N. Laukhin, R. Rousseau, E. Canadell, L. Brossard, D. Jérome, and N.D. Kushch, J. Phys. I 5, 1301 (1995).

${ }^{12}$ H. Weiss, M.V. Kartsovnik, W. Biberacher, E. Steep, E. Balthes, A.G.M. Jansen, K. Andres, and N.D. Kushch, Phys. Rev. B 59, 12370 (1999).

${ }^{13}$ T. Biggs, A.-K. Klehe, J. Singleton, D. Bakker, J. Symington, P. Goddard, A. Ardavan, W. Hayes, J.A. Schlueter, T. Sasaki, and M. Kurmoo, J. Phys.: Condens. Matter 14, L495 (2002).

${ }^{14}$ Y. Yamauchi, M.V. Kartsovnik, T. Ishiguro, M. Kubota, and G. Saito, J. Phys. Soc. Jpn. 65, 354 (1996).

${ }^{15}$ N. Hanasaki, S. Kagoshima, N. Miura, and G. Saito, Phys. Rev. B 63, 245116 (2001)

${ }^{16}$ S. Wanka, J. Hagel, D. Beckmann, J. Wosnitza, J.A. Schlueter, J.M. Williams, P.G. Nixon, R.W. Winter, and G.L. Gard, Phys. Rev. B 57, 3084 (1998).

${ }^{17}$ Unless otherwise stated, the $T_{c}$ values in this paper are extracted from the resistive midpoints.

${ }^{18}$ J. Müller, M. Lang, F. Steglich, J.A. Schlueter, A.M. Kini, U. Geiser, J. Mohtasham, R.W. Winter, G.L. Gard, T. Sasaki, and N. Toyota, Phys. Rev. B 61, 11739 (2000).
${ }^{19}$ I. Olejniczak, B.R. Jones, Z. Zhu, J. Dong, J.L. Musfeldt, J.A. Schlueter, E. Morales, U. Geiser, P.G. Nixon, R.W. Winter, and G.L. Gard, Chem. Mater. 11, 3160 (1999).

${ }^{20}$ B.H. Ward, J.A. Schlueter, U. Geiser, H.H. Wang, E. Morales, J.P. Parakka, S.Y. Thomas, J.M. Williams, P.G. Nixon, R.W. Winter, G.L. Gard, H.-J. Koo, and M.-H. Whangbo, Chem. Mater. 12, 343 (2000).

${ }^{21}$ M. Calandra, J. Merino, and R.H. McKenzie, Phys. Rev. B 66, 195102 (2002).

${ }^{22}$ J. Merino and R.H. McKenzie, Phys. Rev. Lett. 87, 237002 (2001).

${ }^{23}$ U. Geiser, J.A. Schlueter, H.H. Wang, A.M. Kini, J.M. Williams, P.P. Sche, H.I. Zakowicz, M.L. VanZile, and J.D. Dudek, J. Am. Chem. Soc. 118, 9996 (1996).

${ }^{24}$ T.F. Smith, C.W. Chu, and M.B. Maple, Cryogenics 9, 53 (1969).

${ }^{25}$ Since after changing $p$, the pressure cell containing the sample was immersed into the cold sample space inside the cryostat, reliable resistance data could be obtained only below about 250 $\mathrm{K}$.

${ }^{26}$ M.-S. Nam, A. Ardavan, J.A. Symington, J. Singleton, N. Harrison, C.H. Mielke, J.A. Schlueter, R.W. Winter, and G.L. Gard, Phys. Rev. Lett. 87, 117001 (2001).

${ }^{27}$ X. Su, F. Zuo, J.A. Schlueter, M.E. Kelly, and J.M. Williams, Phys. Rev. B 57, R14 056 (1998).

${ }^{28}$ J. Wosnitza, J. Hagel, J.S. Qualls, J.S. Brooks, E. Balthes, D. Schweitzer, J.A. Schlueter, U. Geiser, J. Mohtasham, R.W. Winter, and G.L. Gard, Phys. Rev. B 65, 180506(R) (2002).

${ }^{29}$ K. Kadowaki and S.B. Woods, Solid State Commun. 58, 507 (1986).

${ }^{30}$ O. Stockert and J. Hagel (unpublished).

${ }^{31}$ D. Beckmann, S. Wanka, J. Wosnitza, J.A. Schlueter, J.M. Williams, P.G. Nixon, R.W. Winter, G.L. Gard, J. Ren, and M.-H. Whangbo, Eur. Phys. J. B 1, 295 (1998).

${ }^{32}$ J. Wosnitza, S. Wanka, J.S. Qualls, J.S. Brooks, C.H. Mielke, N. Harrison, J.A. Schlueter, J.M. Williams, P.G. Nixon, R.W. Winter, and G.L. Gard, Synth. Met. 103, 2000 (1999).

${ }^{33}$ J. Wosnitza, S. Wanka, J. Hagel, E. Balthes, N. Harrison, J.A. Schlueter, A.M. Kini, U. Geiser, J. Mohtasham, R.W. Winter, and G.L. Gard, Phys. Rev. B 61, 7383 (2000); J. Hagel, S. Wanka, J. Wosnitza, E. Balthes, J.A. Schlueter, A.M. Kini, U. Geiser, J. Mohtasham, R.W. Winter, and G.L. Gard, Synth. Met. 120, 813 (2001).

${ }^{34}$ D. Shoenberg, Magnetic Oscillations in Metals (Cambridge University Press, Cambridge, 1984).

${ }^{35}$ Since for the present material the exact compressibiliy is unknown we used the value for $\kappa$-(BEDT-TTF $)_{2} \mathrm{Cu}(\mathrm{NCS})_{2}$ typical 
for organic metals: D. Chasseau, J. Gaultier, M. Rahal, L. Ducasse, M. Kurmoo, and P. Day, Synth. Met. 41-43, 2039 (1991).

${ }^{36}$ H. Tanino, K. Kato, M. Tokumoto, H. Anzai, and G. Saito, J. Phys. Soc. Jpn. 54, 2390 (1985).

${ }^{37}$ J. Hagel, J. Wosnitza, C. Pfleiderer, J.A. Schlueter, U. Geiser, J. Mohtasham, R.W. Winter, and G.L. Gard, Synth. Met. 137, 1267 (2003).

${ }^{38}$ K. Yamaji, J. Phys. Soc. Jpn. 58, 1520 (1989).

${ }^{39}$ J. Wosnitza, Fermi Surfaces of Low-Dimensional Organic Metals and Superconductors (Springer, Berlin, 1996).

${ }^{40}$ For the temperature and field range where the $\mathrm{SdH}$ signals were investigated under pressure, $\Delta \sigma / \sigma$ was always small enough so that the determination of the exact background resistance was of negligible influence on the presented results. This is in contrast to the high-field case at $p=0$ discussed in J. Wosnitza, S. Wanka, J. Hagel, H.V. Löhneysen, J.S. Qualls, J.S. Brooks, E.
Balthes, J.A. Schlueter, U. Geiser, J. Mohtasham, R.W. Winter, and G.L. Gard, Phys. Rev. Lett. 86, 508 (2001).

${ }^{41}$ R.H. McKenzie, cond-mat/9905044 (unpublished).

${ }^{42}$ W. Kohn, Phys. Rev. 123, 1242 (1961).

${ }^{43}$ R.S. Edwards, J.A. Symington, E. Rzepniewski, A. Ardavan, J. Singleton, and J.A. Schlueter, Synth. Met. 120, 1033 (2001).

${ }^{44}$ R.H. McKenzie and P. Moses, Phys. Rev. B 60, 11241 (1999).

${ }^{45}$ P.B. Allen and R.C. Dynes, Phys. Rev. B 12, 905 (1975).

${ }^{46}$ For $\lambda_{e e} \neq 0$ the resulting fit parameter $m_{b}$ simply has to be scaled by $\left(1+\lambda_{e e}\right)^{-1}$.

${ }^{47}$ H. Elsinger, J. Wosnitza, S. Wanka, J. Hagel, D. Schweitzer, and W. Strunz, Phys. Rev. Lett. 84, 6098 (2000).

${ }^{48}$ The phenomenological description of the specific-heat data actually gives an $\langle\omega\rangle$ which is about half the value estimated here.

${ }^{49}$ R.W. Stark and C.B. Friedberg, J. Low Temp. Phys. 14, 111 (1974). 